\title{
Comparing the surgical outcomes of stapled anastomosis versus hand-sewn anastomosis of duodenojejunostomy in pylorus-preserving pancreaticoduodenectomy
}

\author{
Dong-Hwan Kim ${ }^{1}$, Soon-Chan Hong ${ }^{1}$, Jae-Yool Jang ${ }^{2}$, Jin-Kyu Cho ${ }^{1}$, Young-Tae Ju', \\ Young-Joon Lee ${ }^{1}$, Eun-Jung Jung', Sang-Ho Jeong', Tae-Jin Park ${ }^{2}$, Ju-Yeon Kim ${ }^{1}$, \\ Seung-Jin Kwag ${ }^{1}$, Ji-Ho Park', and Chi-Young Jeong
}

Department of General Surgery, ${ }^{1}$ Gyeongsang National University Hospital, Gyeongsang National University School of Medicine, Jinju, ${ }^{2}$ Gyeongsang National University Changwon Hospital, Gyeongsang National University School of Medicine, Changwon, Korea

\begin{abstract}
Backgrounds/Aims: This study is to evaluate the perioperative outcomes of the circular stapled method versus the hand-sewn method of the duodenojejunostomy (DJ) procedure in pylorus preserving pancreaticoduodenectomy (PPPD). Methods: In this study, as noted between 2010 and 2018, there were 77 PPPDs which were performed at our hospital by one surgeon. We began the circular stapled method from 2014, and continue with this procedure for the aforementioned surgeries including and up to today. The clinical data for the study were collected retrospectively to compare clinical outcomes of the two methods, the circular stapled anastomosis and the hand - sewn anastomosis. Results: There were 34 patients in a circular stapled group, and 43 in a hand-sewn group as identified for this study. The delayed gastric emptying (DGE) occurred in $6(17.64 \%)$ patients in the circular stapled group, and $10(23.3 \%)$ in the hand-sewn group $(p=0.547)$. It is noted that there was a serum albumin level measured on the 14th day after the operation, which was significantly high in the circular stapled group $(3.41 \pm 0.47$ (g/dl) vs $2.92 \pm 0.39$ (g/dl), $p<0.001)$. There were no significant differences in terms of the incidence of postoperative complications $(58.8 \%$ vs $58.1 \%, p=0.952)$ and mortality rates $(5.9 \%$ vs $0, p=0.192)$ among the patient participants in this study. Conclusions: We conclude that using a circular stapler for the DJ procedure in PPPDs do not increase the development of a DGE, and is also helpful for the benefit of the patient's nutritional status going forward during recovery from the operation. (Ann Hepatobiliary Pancreat Surg 2019;23:245-251)
\end{abstract}

Key Words: Delayed gastric emptying; Pylorus preserving pancreaticoduodenectomy; Stapled anastomosis; Hand-sewn anastomosis; Duodenojejunostomy

\section{INTRODUCTION}

Since the first introduction of the stapling instruments, many surgeons have performed alimentary reconstruction using stapler. ${ }^{1}$ A number of previous researchers reported the stability and usefulness of stapled anastomosis in upper gastrointestinal and colorectal surgeries. ${ }^{2,3}$ Nevertheless, in conventional pylorus-preserving pancreaticoduodenectomy (PPPD), hand-sewn anastomosis was still used for duodenojejunostomy (DJ). ${ }^{4}$

Many surgeons thought that circular stapled anastomosis would induce delayed gastric emptying (DGE) more than hand-sewn anastomosis because of the small diameter of the anastomosis site and disrupted intestinal neural connections. ${ }^{5}$ Several previous researchers have reported on stapled anastomosis of gastro/duodenojejunostomy in pancreatoduodenectomy (PD). Sakamoto et al. ${ }^{6,7}$ introduced Roux-en-Y reconstruction using a circular stapler (CS), and showed that it was an acceptable option for DJ in PPPD. Other research showed that the stapler was faster in gastro/duodenojejunostomy than the hand-sewn procedure. ${ }^{8}$ However, no authors have reported the use of sta-

Received: February 7, 2019; Revised: April 21, 2019; Accepted: April 30, 2019

Corresponding author: Chi-Young Jeong

Department of General Surgery, Gyeongsang National University Hospital, Gyeongsang National University School of Medicine, 79 Gangnam-ro, Jinju 52727, Korea

Tel: +82-55-750-8447, Fax: +82-55-757-5442, E-mail: drjcy@hanmail.net

Copyright (C) 2019 by The Korean Association of Hepato-Biliary-Pancreatic Surgery

This is an Open Access article distributed under the terms of the Creative Commons Attribution Non-Commercial License (http://creativecommons.org/ licenses/by-nc/4.0) which permits unrestricted non-commercial use, distribution, and reproduction in any medium, provided the original work is properly cited. Annals of Hepato-Biliary-Pancreatic Surgery • pISSN: 2508-5778 - eISSN: 2508-5859 
pled anastomosis with duodenojejunostomy limited to PPPD alone. In this study, we introduce the use of end-to-side DJ using a circular stapler, and we retrospectively compared the surgical outcomes with those from hand-sewn anastomosis in PPPD.

\section{PATIENTS AND METHODS}

Through our electronic medical records, we reviewed patients who underwent a pancreaticoduodenectomy (PD) between January 2010 and May 2018. Of the 138 patients chosen, we excluded those who underwent a Whipple operation, subtotal stomach-preserving pancreaticoduodenectomy, or a total PD. Following their elimination, there were 77 eligible patients who underwent PPPD, and their diagnoses were pancreatic cancer $(n=13)$, bile duct cancer $(n=32)$, Vater or duodenal cancer $(n=30)$, or other disease $(\mathrm{n}=2)$. We began using CS anastomosis in 2014 and performed 34 procedures; 43 patients underwent hand-sewn DJ. This retrospective study was approved by the Institutional Review Board (GNUH 2018-07-019).

\section{PPPD}

The specifics of the surgical procedure of PPPD before anastomosis are well-known in the medical community ${ }^{4}$ : After specimens are removed-the common bile duct, pan- creas head, and duodenum - the jejunal limb is lifted through the mesocolon right to the middle colic artery. We performed end-to-side, duct-to-mucosa, two-layer pancreaticojejunostomy (PJ) with an interrupted suture using 5-0 absorbable monofilament thread. All patients had an external pancreatic duct stent with a 3-8 Fr catheter affixed for the proper drainage of the pancreatic juice. After PJ, we performed hepaticojejunostomy (HJ) at the same jejunal loop in single-layered end-to-side configuration using an interrupted suture with a 4-0 polyglycolic-acid. In these cases, the stent was not placed at the HJ.

\section{Duodenojejunostomy}

When we resected the duodenal bulb by a linear stapler (DST Series $^{\mathrm{TM}}$ GIA $^{\mathrm{TM}} 60 \mathrm{~mm}$, Covidien), we preserved more than $3 \mathrm{~cm}$ of duodenal bulb from the pyloric ring in both the circular stapler anastomosis group (CS group) and the hand-sewn anastomosis group (HS group).

The hand-sewn anastomosis was performed by two-layer Albert-Lambert method in an antecolic configuration. The Braun anastomosis was not performed. To decompress anastomosis site, the nasogastric tube was summarily placed into the afferent jejunal limb through the anastomosis.

The stapled anastomosis was performed using a circular stapler $\left(\mathrm{EEA}^{\mathrm{TM}}\right.$ stapler with DST Series ${ }^{\mathrm{TM}} 28 \mathrm{~mm}$ Single
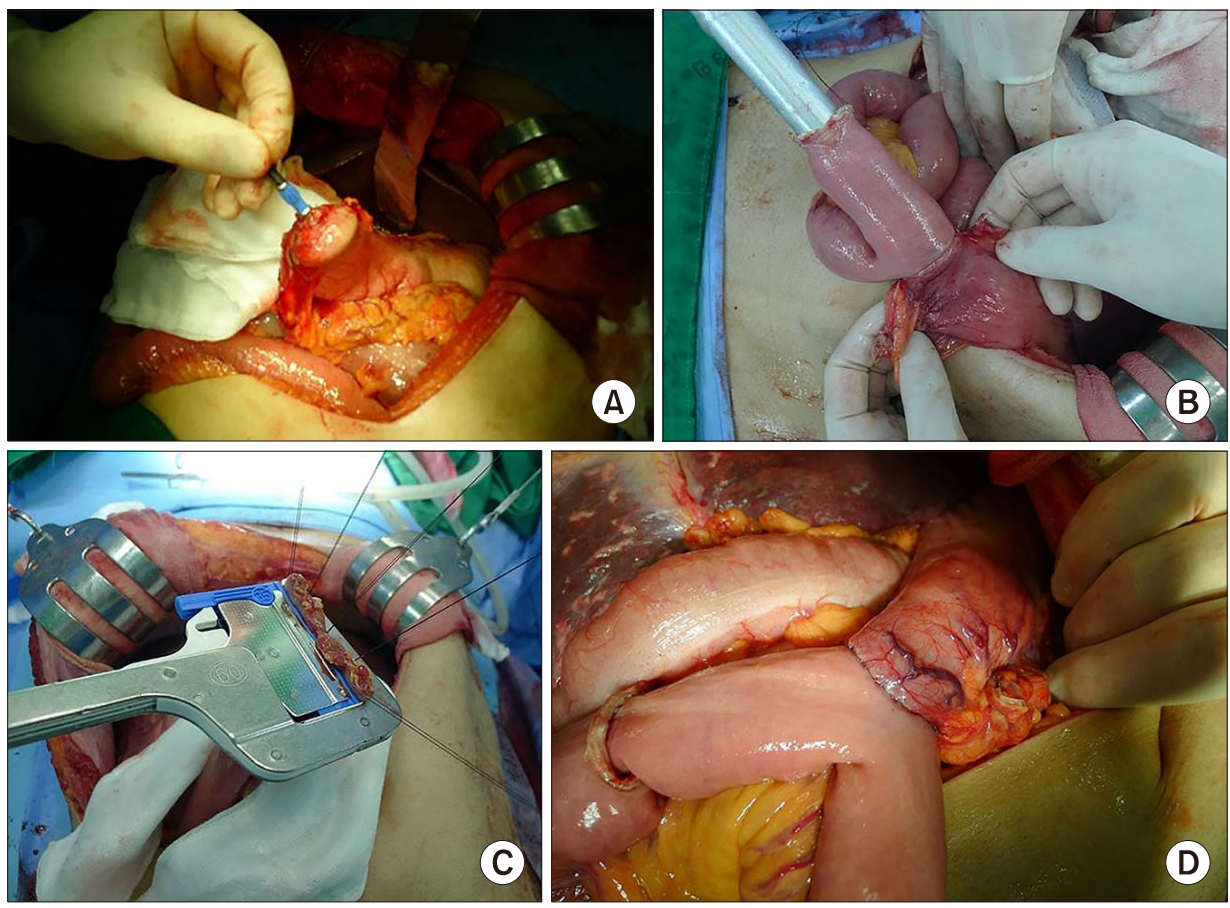

Fig. 1. Stepwise photo of the stapled duodenojejunostomy. (A) Anvil is inserted to the duodenal stump. (B) The circular stapler is inserted into the afferent jejunal limb through a transversely incised opening. (C) The opening of the afferent jejunal limb is closed using a linear stapler. (D) The completion of the stapled duodenojejunostomy. 
Use Stapler, Covidien, Dublin, Ireland). During this procedure, an anvil was inserted into the duodenal stump and fixed with a purse-string suture (Fig. 1A). Next, a circular stapler was inserted into the afferent jejunal loop through a small transverse enterotomy and connected with the anvil (Fig. 1B). After the anastomosis was complete, the enterotomy at the jejunum was closed with a linear stapler (DST Series ${ }^{\mathrm{TM}} \mathrm{TA}^{\mathrm{TM}} 90 \mathrm{~mm}$, Covidien; Fig. 1C). The length from the DJ anastomosis site to the pyloric ring was enough to conserve the shape and function of the pyloric ring (Fig. 1D). As noted, Braun anastomosis was not performed and a nasogastric tube was placed the same way as the hand-sewn anastomosis that was mentioned earlier. Drains were placed anterior to the PJ site, anterior and posterior to the HJ site. One surgeon performed all procedures.

\section{Postoperative management}

Postoperative management of all patients was standardized in this study. The patients received prophylactic antibiotic up to and until postoperative day 1 and for longer duration when there was evidence of an infection. The octreotide was used routinely to all patients until postoperative day 5. The nasogastric tubes were removed from the patients when the drainage volume was less than 200 $\mathrm{ml} /$ day.

The patients started to take sips of water beginning the day after the nasogastric tube was removed.

The patients were permitted to begin nutrition consisting of a soft bland diet, as taken after take sips of water for 1 day. If patients complained of abdominal discomfort, nausea, or vomiting, we suspended their intake diet and took abdominal radiography to check for the presence of severe stomach distension or ileus. The albumin levels in serum were routinely measured on preoperative and postoperative day 14. If the serum albumin level was lower than $3 \mathrm{~g} / \mathrm{dl}$ on the routine laboratory exam, we used 100 $\mathrm{ml}$ of $20 \%$ albumin intravenously for the supplement, with a maximum dosage of twice a week. The amylase and lipase levels in the patients' drained fluid were routinely measured on postoperative day 3 and 5, and abdominal computed tomography (CT) scans were taken on postoperative day 7. Later, patients' drains were removed when there was no sign of a pancreatic fistula, intra-abdominal fluid collection, or other operation-related findings on CT. Total parenteral nutrition for each patient was administered when the patients needed long-term fasting or did not eat until postoperative day 5 .

\section{Definition of outcome measures}

In this study, we compared the baseline characteristics, intraoperative factors, and surgical outcomes between the CS group and the HS group. In these terms, we defined delayed gastric emptying (DGE) according to International Study Group of Pancreatic Surgery (ISGPS) criteria ${ }^{9}$ : duration of nasogastric tube placement in the patient, patient's postoperative condition on a day when the nasogastric tube is reinserted, postoperative time during which the patient cannot tolerate solid oral intake, presence of vomiting or gastric distension, or use of prokinetics to improve the patient's condition postoperatively (Table 1). In this study, we classified postoperative complications according to the Clavien-Dindo classification and were defined clinically significant when classified as grade III or more. We classified postoperative pancreatic fistula as ISGPS grade $\mathrm{A}, \mathrm{B}$, or $\mathrm{C}$ according to the criteria ${ }^{10}$ and grade $\mathrm{B}$ and $\mathrm{C}$ were defined clinically significant in this study. To assess patients' nutritional status, serum albumin level was measured preoperatively and on postoperative day 14 .

\section{Statistical analysis}

We performed the statistical analyses for this study us-

Table 1. Delayed gastric emptying grade of international study group of pancreas surgery

\begin{tabular}{clccc}
\hline DGE grade & NGT required & $\begin{array}{c}\text { Unable to tolerate solid } \\
\text { oral intake by POD }\end{array}$ & $\begin{array}{c}\text { Vomiting/gastric } \\
\text { distension }\end{array}$ & $\begin{array}{c}\text { Use of } \\
\text { prokinetics }\end{array}$ \\
\hline A & 4-7 days or reinsertion > POD3 & 7 & \pm & \pm \\
B & $8-14$ days or reinsertion > POD7 & 14 & + & + \\
C & $>14$ days or reinsertion > POD14 & 21 & + & + \\
\hline
\end{tabular}

DGE, delayed gastric emptying; POD, postoperative day; NGT, nasogastric tube 
ing SPSS Statistics 21. We analyzed the categorical variables using Fisher's exact probability test or chi-square test and the continuous variables using the Student $t$ test to compare the two groups. We considered $\mathrm{p}$ less than 0.05 statistically significant for the study purposes.

\section{RESULTS}

\section{Patient characteristic}

In this study, a total of 77 patients underwent PPPD, 34 in the CS group and 43 in the HS group; the patient characteristics are shown in Table 2. The CS group was older than the HS group (mean age: $68.5 \pm 7.07$ vs $62.1 \pm 10.8$, $p=0.003$ ), but there were otherwise no significant differences in gender, BMI, American Society of Anesthesiolo-

Table 2. Preoperative characteristics of patients belonged to circular stapler (CS) anastomosis and hand-sewn (HS) anastomosis groups

\begin{tabular}{lccc}
\hline & CS group $(\mathrm{n}=34)$ & HS group $(\mathrm{n}=43)$ & $p$-value \\
\hline Age $(\mathrm{yr})$ & $68.52 \pm 7.07$ & $62.14 \pm 10.8$ & 0.003 \\
Gender $(\mathrm{M} / \mathrm{F})$ & $20 / 14$ & $21 / 22$ & 0.383 \\
BMI $\left(\mathrm{kg} / \mathrm{m}^{2}\right)$ & $22.61 \pm 2.36$ & $22.34 \pm 3.00$ & 0.663 \\
ASA & & & 0.598 \\
1 & 0 & 0 & \\
2 & 25 & 34 & 0.059 \\
3 & 9 & $24(55.8 \%)$ & 0.036 \\
Preoperative biliary drainage & $26(76.5 \%)$ & $15(34.9 \%)$ & 0.579 \\
Diabetes & $20(58.8 \%)$ & $3.60 \pm 0.49$ & 0.022 \\
Preoperative serum albumin level $(\mathrm{g} / \mathrm{dl})$ & $3.53 \pm 0.57$ & & \\
Diagnosis & $9(26.5 \%)$ & $22(51.2 \%)$ & \\
Pancreatic cancer & $8(23.5 \%)$ & $15(34.9 \%)$ & 2 \\
Ampullary or duodenal cancer & $17(50.0 \%)$ & 2 & \\
Bile duct cancer & 0 & & \\
Other & &
\end{tabular}

Table 3. Perioperative outcomes in circular stapler (CS) anastomosis and hand-sewn (HS) anastomosis groups

\begin{tabular}{lccc}
\hline & CS group (n=34) & HS group (n=43) & $p$-value \\
\hline Hospital stay (days) & $29 \pm 15$ & $30 \pm 16$ & 0.773 \\
Operative time (min) & $429.3 \pm 59.8$ & $428.5 \pm 65.1$ & 0.957 \\
Intraoperative blood loss (ml) & $833.5 \pm 579.9$ & $1084 \pm 541.1$ & 0.054 \\
Duration of keeping nasogastric tube (days) & $2.7 \pm 2.23$ & $2.7 \pm 2.21$ & 0.904 \\
Resuming liquid oral intake (postoperative day) & $4.5 \pm 2.16$ & $5.81 \pm 1.94$ & 0.007 \\
Starting solid diet (postoperative day) & $7.05 \pm 3.5$ & $7.39 \pm 2.39$ & 0.619 \\
Reinsertion of nasogastric tube & $2(5.9 \%)$ & $3(7.0 \%)$ & 0.428 \\
Decrease in the serum albumin level after operation (g/dl) & $0.12 \pm 0.55$ & $0.67 \pm 0.55$ & $<0.001$ \\
Serum albumin level, postoperative day 14 (g/dl) & $3.41 \pm 0.47$ & $2.92 \pm 0.39$ & $<0.001$ \\
Postoperative complications & $20(58.8 \%)$ & $25(58.1 \%)$ & 0.952 \\
Postoperative pancreatic fistula & $16(47.1 \%)$ & $15(34.9 \%)$ & 0.279 \\
Delayed gastric emptying & $6(17.6 \%)$ & $10(23.3 \%)$ & 0.547 \\
A & $3(8.8 \%)$ & $5(11.6 \%)$ & \\
B & $3(8.8 \%)$ & $4(9.3 \%)$ & \\
C & 0 & $1(2.3 \%)$ & $0.3 \%$ \\
Anastomotic bleeding & $2(5.9 \%)$ & $0(0 \%)$ & 0.192 \\
Pseudoaneurysm & $4(11.8 \%)$ & $2(4.7 \%)$ & 0.397 \\
Wound problem & $5(14.7 \%)$ & $3(7.0 \%)$ & 0.454 \\
Pneumonia & $1(2.9 \%)$ & $1(2.3 \%)$ & $>0.999$ \\
Mortality & $2(5.9 \%)$ & 0 & 0.192 \\
\hline
\end{tabular}


gists score, preoperative biliary drainage, medical history of diabetes, preoperative serum albumin level, or diagnosis between the two groups

\section{Perioperative outcomes}

Table 3 shows the noted perioperative outcomes in both groups; there were no significant differences in operative time or blood loss between the groups $(429.3 \pm 59.8$ vs $428.5 \pm 65.1$ minutes, $p=0.957 ; 833.5 \pm 579.9$ vs $1084 \pm 541.1$ $\mathrm{ml}, p=0.054)$. Although there was no significant difference in the duration of nasogastric tube placement between the two groups ( $2.7 \pm 2.23$ vs $2.7 \pm 2.21$ days, $p=0.904$ ), the measured time to resume oral fluid ingestion was significantly earlier in the CS group than in the HS group ( $4.5 \pm 2.16$ vs $5.81 \pm 1.94$ days, $p=0.007)$. Relatedly, the incidence of DGE was $17.6 \%$ in the CS group and $23.3 \%$ in the HS group, although the difference was not statistically significant; additionally, the overall incidence of DGE was not higher in the CS group than in the HS group ( $17.6 \%$ vs $23.3 \%, p=0.547$ ), although notably, serum albumin on postoperative day 14 was significantly higher in the CS group $(3.41 \pm 0.47$ vs $2.92 \pm 0.39 \mathrm{~g} / \mathrm{dl}, p<0.001)$. Furthermore, the noted decrease in the serum albumin level after the operation was also significantly lower in the CS group than in the HS group $(0.12 \pm 0.55$ vs $0.67 \pm .055$ $\mathrm{g} / \mathrm{dl}, p<0.001)$. There were no differences in frequency of albumin supplementation $(3.32 \pm 3.27$ vs $3.48 \pm 3.67$ times, $p=0.836)$, postoperative complication rates $(58.8 \%$ vs $58.1 \%$, $p=0.952$ ), postoperative pancreatic fistula (34.9 vs 47.1, $p=0.279$ ), duration of hospital stays ( $29 \pm 15$ vs $30 \pm 16, p=$ $0.773)$, or mortality rates $(5.9 \%$ vs $0, p=0.192)$ between the two groups.

\section{DISCUSSION}

In this study, we compared the clinical outcomes of circular stapler and conventional hand-sewn anastomosis for DJ in a total of 77 patients who underwent PPPD. We confirmed through this study that the incidence of DGE was not higher in the CS group than in the HS group; the albumin level on postoperative day 14 was markedly higher in the CS group; and there were no differences in postoperative complication rates or 30-day mortality between the two patient groups.

It is emphasized that DGE is one of the most common complications of PPPD, occurring in $19-57 \%$ of patients, ${ }^{9}$ but its pathogenesis is still unclear. Several factors have been reported to affect DGE in previous studies, including intraabdominal complications, ${ }^{5}$ gastric atony due to reduced motilin levels, ${ }^{11}$ pylorospasm secondary to vagotomy, ${ }^{12}$ angulation of the reconstructed alimentary tract, ${ }^{13}$ and reconstruction type. ${ }^{6,14}$ Nevertheless, the obvious fact to note is that there are significant issues with the de-vascularization and denervation of the pyloric regions. ${ }^{12,15}$ For this reason, a stapled anastomosis has theoretical advantages, such as that it does not require clamping of the intestinal tube, which is necessary during hand-sewn procedures, and it creates a single layer of anastomosis, whereas the hand-sewn method requires a double layer. These differences may cause fewer incidences of bowel edema and better blood supply to the region, which are favorable for decreasing the incidence of DGE. ${ }^{16,17}$

Other researchers have compared stapled anastomosis with hand-sewn anastomosis. ${ }^{6,17,18}$ Sakamoto et al. ${ }^{6}$ (2016) have reported that Roux-en-Y reconstruction using a stapler in PD was not inferior to the hand-sewn method in terms of incidence of DGE, and we found favorable results of stapled anastomosis for DJ in PPPD in terms of DGE incidence.

Broadly speaking, serum albumin levels are influenced by many clinical phenomena which include chronic disease, inflammation, using steroids, and acute illness other than the patient's nutritional status. Despite these limitations, many clinicians view albumin levels as synonymous with nutritional status, and the patient albumin levels are often used as a nutritional index in clinical studies. ${ }^{19-21}$ For this study, we measured preoperative and postoperative serum albumin to assess nutritional status. We found that the albumin level on postoperative day 14 was significantly higher in the CS group than in the HS group $(3.41 \pm 0.47$ vs $2.92 \pm 0.39 \mathrm{~g} / \mathrm{dl}, p<0.01)$, and the decrease in albumin level after surgery was significantly lower in the CS group than in the HS group $(0.12 \pm 0.55$ vs $0.67 \pm$ $0.55 \mathrm{~g} / \mathrm{dl}, p<0.001)$. We also compared postoperative albumin levels in the DGE and non-DGE groups, and the albumin level was significantly lower in the DGE group $(2.94 \pm 0.39$ vs $3.19 \pm 0.50 \mathrm{~g} / \mathrm{dl}, p=0.047)$. Hence, it can be inferred that the better postoperative nutritional status observed in the CS group in this study was partially attributable to the lower incidence of DGE.

Previous researchers have reported that use of staplers 
for anastomosis does not increase postoperative complications in $\mathrm{PD},{ }^{6-8,14,17,18,22}$ and we also observed this as well in the present study. The prevailing discipline notes that staple alimentary reconstruction is now widely used in upper and lower gastrointestinal tract surgery. ${ }^{3,23}$ We think that staplers can also be used safely for duodenojejunostomy in PPPD.

In the CS group, there were two cases of anastomosis site bleeding (5.9\%) and mortality (5.9\%) respectively, although the difference in incidence with the HS group was not considered to be statistically significant $(p=0.192)$.

In this case, there are two cases of mortality, one is noted as gastroduodenal artery (GDA) stump bleeding, another is a case of intra-abdominal bleeding. In the first case, the patient was discharged on postoperative day 17 , and again visited the emergency room with abdominal pain. The patient was performed with an angiographic embolization, due to a GDA stump bleeding as noted on the CT findings. However, the re-bleeding occurred after embolization, and as result the patient was expired due to hemorrhagic shock. Subsequently, in the second case, we inserted a percutaneous drainage catheter by radiologic intervention for intraabdominal fluid collection on postoperative day 6. During the procedure, there was the incidence of a massive bleeding due to a vascular injury. Therefore, the patient fell into hemorrhagic shock and was expired on postoperative day 16 . We considered both cases were not related to using a circular stapler.

In the both cases of anastomotic bleeding, a hematoma was drained through a nasogastric tube on postoperative day 1. But since postoperative day 2, the hematoma was not drained with the patient's hemoglobin level was stable so did not require any transfusion overall. In some studies, it is reported that there was a high risk of anastomotic bleeding at using stapler in PD., ${ }^{7,}$ In our study, although it was not statistically significant, it was true that anastomotic bleeding has occurred. Therefore, when using the stapler, we recommend that the surgeon should examine the lumen thoroughly for any instance of anastomotic bleeding.

This study has some limitations other than being a retrospective observatory study. All of the operations were performed by one surgeon, and therefore, more surgery experiences from other surgeons is needed before the results from this study can be generalized; for instance, we did not report the cost of the stapler procedure for this operation. Finally, we did not measure the time taken to perform DJ for each patient, although we could show that there was no difference in terms of total operation time between the two groups.

Notably, in this study, we compared the surgical outcomes between circular anastomosis and hand-sewn anastomosis of duodenojejunostomy in PPPD. Although there were no significant differences in the incidence of delayed gastric emptying and postoperative complication, operation time in both groups, using a circular stapler partially affects the improvement of the patient's nutritional status who underwent PPPD. Therefore, using a circular stapler for DJ in PPPD can be one option. Future studies that reflect on the cost and reconstruction time for DJ in PPPD are needed in the future.

\section{REFERENCES}

1. Ravitch MM, Steichen FM. A stapling instrument for end-to-end inverting anastomoses in the gastrointestinal tract. Ann Surg 1979; 189:791-797.

2. Korolija D. The current evidence on stapled versus hand-sewn anastomoses in the digestive tract. Minim Invasive Ther Allied Technol 2008; 17:151-154.

3. Nomura S, Sasako M, Katai H, Sano T, Maruyama K. Decreasing complication rates with stapled esophagojejunostomy following a learning curve. Gastric Cancer 2000;3:97-101.

4. Yamaguchi K. Pancreatoduodenectomy for bile duct and ampullary cancer. J Hepatobiliary Pancreat Sci 2012;19:210-215.

5. van Berge Henegouwen MI, van Gulik TM, DeWit LT, Allema JH, Rauws EA, Obertop H, et al. Delayed gastric emptying after standard pancreaticoduodenectomy versus pylorus-preserving pancreaticoduodenectomy: an analysis of 200 consecutive patients. J Am Coll Surg 1997;185:373-379.

6. Sakamoto Y, Hori S, Oguro S, Arita J, Kishi Y, Nara S, et al. Delayed gastric emptying after stapled versus hand-sewn anastomosis of duodenojejunostomy in pylorus-preserving pancreaticoduodenectomy: a randomized controlled trial. J Gastrointest Surg 2016;20:595-603.

7. Sakamoto Y, Kajiwara T, Esaki M, Shimada K, Nara S, Kosuge T. Roux-en-Y reconstruction using staplers during pancreaticoduodenectomy: results of a prospective preliminary study. Surg Today 2009;39:32-37.

8. Sato N, Yabuki K, Kohi S, Mori Y, Minagawa N, Tamura T, et al. Stapled gastro/duodenojejunostomy shortens reconstruction time during pylorus-preserving pancreaticoduodenectomy. World J Gastroenterol 2013;19:9399-9404.

9. Wente MN, Bassi C, Dervenis C, Fingerhut A, Gouma DJ, Izbicki JR, et al. Delayed gastric emptying (DGE) after pancreatic surgery: a suggested definition by theInternational Study Group of Pancreatic Surgery (ISGPS). Surgery 2007;142:761-768.

10. Bassi C, Dervenis C, Butturini G, Fingerhut A, Yeo C, Izbicki $\mathrm{J}$, et al.; International Study Group on Pancreatic Fistula Definition. Postoperative pancreatic fistula: an international study group (ISGPF) definition. Surgery 2005;138:8-13. 
11. Ohwada S, Satoh Y, Kawate S, Yamada T, Kawamura O, Koyama $\mathrm{T}$, et al. Low-dose erythromycin reduces delayed gastric emptying and improves gastric motility after Billroth I pylorus-preserving pancreaticoduodenectomy. Ann Surg 2001;234:668-674.

12. Kim DK, Hindenburg AA, Sharma SK, Suk CH, Gress FG, Staszewski $\mathrm{H}$, et al. Is pylorospasm a cause of delayed gastric emptying after pylorus-preserving pancreaticoduodenectomy? Ann Surg Oncol 2005;12:222-227.

13. Ueno T, Tanaka A, Hamanaka Y, Tsurumi M, Suzuki T. A proposal mechanism of early delayed gastric emptying after pylorus preserving pancreatoduodenectomy. Hepatogastroenterology 1995; 42:269-274.

14. Mohammed S, Van Buren Ii G, McElhany A, Silberfein EJ, Fisher WE. Delayed gastric emptying following pancreaticoduodenectomy: incidence, risk factors, and healthcare utilization. World J Gastrointest Surg 2017;9:73-81.

15. Liberski SM, Koch KL, Atnip RG, Stern RM. Ischemic gastroparesis: resolution after revascularization. Gastroenterology 1990; 99:252-257.

16. Itani KM, Coleman RE, Meyers WC, Akwari OE. Pylorus-preserving pancreatoduodenectomy. A clinical and physiologic appraisal. Ann Surg 1986;204:655-664.

17. Murata Y, Tanemura A, Kato H, Kuriyama N, Azumi Y, Kishiwada $\mathrm{M}$, et al. Superiority of stapled side-to-side gastrojejunostomy over conventional hand-sewn end-to-side gastrojejunostomy for reducing the risk of primary delayed gastric emptying after subtotal stomach-preserving pancreaticoduodenectomy. Surg Today 2017;47:1007-1017.

18. Hajibandeh S, Hajibandeh S, Khan RMA, Malik S, Mansour M, Kausar A, et al. Stapled anastomosis versus hand-sewn anastomosis of gastro/duodenojejunostomy in pancreaticoduodenectomy: a systematic review and meta-analysis. Int J Surg 2017; 48:1-8.

19. Covinsky KE, Covinsky MH, Palmer RM, Sehgal AR. Serum albumin concentration and clinical assessments of nutritional status in hospitalized older people: different sides of different coins? J Am Geriatr Soc 2002;50:631-637.

20. Herrmann FR, Safran C, Levkoff SE, Minaker KL. Serum albumin level on admission as a predictor of death, length of stay, and readmission. Arch Intern Med 1992;152:125-130.

21. Klein S. The myth of serum albumin as a measure of nutritional status. Gastroenterology 1990;99:1845-1846.

22. Sakamoto Y, Yamamoto Y, Hata S, Nara S, Esaki M, Sano T, et al. Analysis of risk factors for delayed gastric emptying (DGE) after 387 pancreaticoduodenectomies with usage of 70 stapled reconstructions. J Gastrointest Surg 2011;15:1789-1797.

23. Hansen O, Schwenk W, Hucke HP, Stock W. Colorectal stapled anastomoses. Experiences and results. Dis Colon Rectum 1996; 39:30-36 